\title{
Survival of metastatic colorectal cancer patients treated with chemotherapy in Alberta (1995-2004)
}

\author{
Yiqun Chen • Zhenguo Qiu • Anmmd Kamruzzaman • \\ Tom Snodgrass • Andrew Scarfe • Heather E. Bryant
}

Received: 20 October 2008 / Accepted: 21 April 2009 /Published online: 15 May 2009

(C) The Author(s) 2009. This article is published with open access at Springerlink.com

\begin{abstract}
Goals of work Clinical trials have suggested that advances in chemotherapy significantly improve the survival of patients with metastatic colorectal cancer. Comparable evidence from clinical practice is scarce. This study aims to investigate the survival of patients with metastatic colorectal cancer treated with chemotherapy in Alberta, Canada.

Patients and methods Trends of relative survival of patients diagnosed in 1994-2003 were assessed using Alberta Cancer Registry (ACR) data. The median overall survival (OS) of patients diagnosed in 2004 was determined by linking Cancer Registry data with Electronic Medical Records (EMR). Cox regression models were fitted to calculate the hazard ratio for patients treated with chemotherapy.

Results The 2-year relative survival for patients with metastatic colorectal cancer who received chemotherapy increased significantly from $29 \%$ to $41 \%$ over the 10 years
\end{abstract}

\footnotetext{
Y. Chen $(\bowtie)$
}

Cancer Information and Registries Division,

Cancer Institute New South Wales,

Level 1 Biomedical Building, Australia Technology Park,

Eveleigh, NSW 2015, Australia

e-mail: yiqun.chen@cancerinstitute.org.au

Z. Qiu $\cdot$ A. Kamruzzaman $\cdot$ T. Snodgrass

Division of Population Health and Information,

Alberta Cancer Board,

Edmonton, AB, Canada

\author{
A. Scarfe \\ Cross Cancer Institute, Alberta Cancer Board, \\ Edmonton, AB, Canada \\ H. E. Bryant \\ Canadian Partnership Against Cancer, \\ Toronto, ON, Canada
}

(1994-2003, $p<0.015)$. A $69 \%$ reduction in the risk of mortality was observed in the 168 patients who received chemotherapy compared to the 87 patients who did not, after adjusting for age, gender, and number of metastases. The median OS of patients who received chemotherapy was 17.5 months. This is comparable to the 18-20 months seen in recently published clinical trials, considering the patients in this study were from the real clinical practice, nearly half of them were older than 70 , and many of them might have important co-morbidities.

Conclusions The survival of patients diagnosed with metastatic colorectal cancer in Alberta has improved in recent years; this is most likely attributable in large part to the use of chemotherapy.

Keywords Metastatic colorectal cancer - Chemotherapy . Overall survival

\section{Introduction}

Colorectal cancer is the second leading cause of cancer death in both Alberta and the Western world [1]. The 5-year survival rate for colorectal cancer cases in Alberta was estimated to be $59 \%$ ( $95 \%$ confidence interval $(\mathrm{CI})=57$ $61 \%$ ); this was similar to the rate for all of Canada, $61 \%$ (95\% CI $=60-62 \%$ ) [2]. A continual downward trend in the colorectal cancer mortality rate was observed between 1994 and 2003 in Canada despite a fairly stable incidence rate during this same 10-year period. It has been suggested that this decrease in mortality reflects improvements in treatment, particularly chemotherapy [3]. Screening for colorectal cancer can also reduce both incidence and mortality. However, screening was not likely responsible for this observed reduction in colorectal cancer mortality as the 
National Recommendation for Screening for Colorectal Cancer was first published in 2001 [4] and the estimated screening rate in Alberta had been low in 2004 [5]. The apparent impact of chemotherapy on the decreasing mortality rates of colorectal cancer patients in Alberta requires a more detailed assessment.

Survival after a diagnosis of colorectal cancer is largely determined by whether the cancer has spread (metastasized) and the extent of this spread at the time of diagnosis. Survival can be predicted by establishing the cancer stage [6]. A study using Surveillance, Epidemiology, and End Results (SEER) data for 1973-1997 showed that the 5-year relative survival rate of colorectal cancer was $5 \%$ for stage IV, $55 \%$ for stage III, $87 \%$ for stage II, and $96 \%$ for stage I [7]. The results from the analysis of more recent SEER data (1991-2000) showed that the 5-year stage-specific survival for colon cancer was $8 \%$ for stage IV, $60 \%$ for stage III, $83 \%$ for stage II, and over $93 \%$ for stage I [8]. Patients diagnosed with stage IV colorectal cancer, in which distant metastasis has occurred, have the worst outcomes. This emphasizes the importance of detecting colorectal cancer earlier, ideally before metastasis occurs. Unfortunately, approximately half of all patients with colorectal cancer will develop metastatic disease. Without treatment, the median OS time is approximately 9 months after the detection of metastases [9]. Evidence from clinical trials has shown that the availability of new active chemotherapy drugs in the treatment of metastatic colorectal cancer patients has greatly improved the prognosis of these patients in recent years. Ten years ago, the treatment was based on one drug only, 5-fluorouracil (5-FU). With the availability of new active drugs and the development of new drug combinations, a patient with metastatic colorectal cancer should be expected to have a median survival of 18 20 months compared to that of 11-14 months just a few years ago $[10,11]$.

Evidence from population-based cancer survival analysis and reviews of clinical trials suggest that advances in chemotherapy can improve the survival of colorectal cancer patients, in particular the OS of those patients with distant metastases. Previous reports have emphasized the importance of linking data from population-based cancer registries and cancer care databases in order to monitor the quality of cancer treatment $[12,13]$. Identification of all cancer diagnoses from a population-based cancer registry, followed by an evaluation of the outcomes, provides an opportunity for the results to be generalized to the total population. Chemotherapy information has been available in Electronic Medical Record (EMR) of the Alberta Cancer Board (ACB) since 2002. Therefore, the linkage between the population-based Cancer Registry data and EMR data for studying treatment outcomes has only recently become possible. The aim of the current study is to investigate the survival of metastatic colorectal cancer patients treated with chemotherapy in Alberta and to assess whether there has been improvement in recent years as was reported in the literature.

\section{Methods}

Patients diagnosed with invasive adenocarcinoma of the colon, rectosigmoid junction, and rectum between 1994 and 2004 were included in this study. Study cases were selected from ACR using a combination of ICD-O topography codes $(\mathrm{C} 18, \mathrm{C} 19$, and $\mathrm{C} 20)$ and morphology codes for adenocarcinoma [14]. Cases of other or unspecified carcinoma, lymphoma, and sarcoma were excluded. Cases confirmed by cytology or histology were included while cases whose diagnoses were confirmed only by clinical examination, radiology, autopsy, or death certificate were excluded. These comprised around $10 \%$ of the overall colorectal cancer cases diagnosed in the study period. Patients with multiple primary cancers were not excluded in order to reflect the patient population. Cases diagnosed with distant metastases at the time of diagnosis were identified.

Cases diagnosed in 2004 were linked with the EMR in the ACB's Varis Medical Oncology Manager Database using the patient's unique identification number. Information about patients' age, gender, diagnosis, metastasis, initial treatment, and last known vital status was extracted from ACR, whereas the patients' cancer center appointment and treatment information was obtained from the EMR. The ACR is a population-based cancer registry that has a proven high level (over 95\%) of completeness for registration of all new cancer incidences and cancer deaths in Alberta, according to the standards of the North American Association of Central Cancer Registries [15]. The Cancer Registry conducts active follow-up every month to obtain the vital status of all cancer patients dying in Alberta through Alberta Vital Statistics. Every year, the Cancer Registry also links with the national vital statistics database to confirm the number of deaths among cancer patients and to identify the vital status of those patients who moved from Alberta to other Canadian provinces. In this way, virtually all patients with metastatic colorectal cancer at diagnosis in Alberta can be identified. Since all chemotherapy treatments for metastatic colorectal cancer patients in Alberta are provided by the cancer treatment centers of the $\mathrm{ACB}$, patients who received chemotherapy can be identified from the EMR system of the ACB.

At the current stage of EMR implementation, there is no single indicator confirming the administration of chemotherapy; however, there are several potential indicators which can be used as surrogates. These include initial treatment data from the Cancer Registry database, appoint- 
ment information for chemotherapy administration, location of the treatment appointments in the EMR, drug dosage recording information, and the name of the chemotherapy regimen used. Although the latter two indicators can provide more specific information concerning the chemotherapy treatment, they were incomplete at the time of this review as the on-line ordering of chemotherapy drugs had not yet been fully implemented. Therefore, in this study, the location and the date of the first chemotherapy appointment were used as indicators for chemotherapy administration.

Patients included in this study were tracked through to September 30, 2006. Two types of survival analysis were carried out. First, the 10-year trends of relative survival were assessed using cases diagnosed between 1994 and 2003; a comparison was made between patients who received chemotherapy as the initial treatment to those who did not. Relative survival is the ratio of the observed survival rate in a group of cancer patients to the expected survival rate for a group of people in the general population with similar gender, age, residence, and calendar time period. Age- and sex-specific mortality rates in the general population were obtained from 1996-2001 life tables provided by Statistics Canada for Albertans. The 5-year survival rate has conventionally been used as an index for comparing survival across groups of patients. However, the choice of survival interval, such as 1 year, 2 years, or 5 years after the diagnosis, will depend on the prognosis of the cancer concerned and the number of individuals (usually requires more than ten patients) entering a survival interval [16]. In this study, 1-year, 2-year, and 5-year relative survival rates with $95 \%$ confidence intervals (CIs) were calculated. In order to stabilize the survival rates, calculations were carried out for cases diagnosed in every 2 years (e.g., diagnosis calendar years 1994-1995 and 2002-2003) rather than in a single year. The relative survival rates between the years 1994 and 1999 were computed using cohort analysis whereas the rates between 2000 and 2003 were predicted rates using period analysis (when complete follow-up data are not available) [17]. The Ederer-II method was used to estimate the expected survival rate [18].

Second, a retrospective survival analysis was conducted by reviewing a cohort of patients diagnosed with metastatic colorectal cancer in 2004. Median OS time and the 95\% CI of these patients were calculated for two follow-up periods with two respective starting dates. The first follow-up was from the first appointment of any treatment to the date of death or the last follow-up; the second follow-up was from the date of the first appointment of chemotherapy to the date of death or the last follow-up. Patients without a death record at the end of follow-up were assumed to be alive. Survival curves were estimated using the Kaplan-Meier method. A log-rank test was performed to determine the statistically significant differences between the survival curves. A Cox regression model is fitted to compare the survival of patients who received chemotherapy compared to those who did not, accounting for potential confounding factors associated with treatment and survival outcomes. These confounding factors included age at diagnosis, gender, site of metastasis, number of metastasis, and having multiple primary cancers. All through the analyses, $p$ values less than 0.05 were considered statistically significant.

Statistical analyses were performed using SAS 9.1 (SAS Institute Inc, Cary, NC, USA). Ethical approval was obtained from the Research Ethics Board of the ACB.

\section{Results}

Between 1994 and 2003, 11,360 Albertans were diagnosed with invasive colorectal cancer and were microscopically confirmed to be adenocarcinoma. Of them, 2,196 (19\%) had distant metastasis at the time of their diagnosis. The proportion of patients with distant metastases was higher in younger patients than in older patients, with $27 \%$ among patients in age $<50,24 \%$ in $50-59,21 \%$ in $60-69,18 \%$ in $70-79$, and $13 \%$ in $80+$. The average and median age of the patients with metastatic colorectal cancer was 66 and 67 years, respectively. A patient may receive more than one treatment (e.g., chemotherapy, surgery, radiation therapy, etc.) according to their initial treatment plan. On average, about $40 \%$ of the patients diagnosed with distant metastasis in 1994-2003 received chemotherapy in their initial treatment. This proportion was doubled, from $26 \%$ in $1994-1995$ to $51 \%$ in $2002-2003$. Table 1 shows the trends of relative survival rates for five cohorts (each cohort contained two calendar years of diagnosis) stratified based upon presence or absence of distant metastasis and whether they received chemotherapy or not. A statistically significant increase in survival rates is observed for patients who received chemotherapy but not for the patients in the nonchemotherapy group. For the patient who had distant metastasis at the time of diagnosis, and received chemotherapy, the 1-year survival rate increased from $63 \%$ in $1994-1995$ to $75 \%$ in $2002-2003(p=0.016)$ and the 2 -year survival rate increased from $29 \%$ to $41 \%$ ( $p=0.015$ ) in the same time period. The 5 -year survival rate also increased for this group, but this increase was not statistically significant $(p=0.122)$.

In 2004, 1,513 Albertans were diagnosed with colorectal cancer. Among them, $378(25 \%)$ had distant metastasis at the time of diagnosis and 374 of the 378 had attended any ACB-affiliated cancer treatment center. The information of these 374 patients was obtained from the EMR of ACB. The four remaining cases were elderly patients who died soon after diagnosis with unknown treatment. In total, 328 
Table 1 One-, 2-, and 5-year relative survival rates \% and (95\% CIs) for patients diagnosed with microscopically verified with adenocarcinoma colorectal cancer in Alberta, 1994-2003

\begin{tabular}{|c|c|c|c|c|c|c|c|c|}
\hline & & $\begin{array}{l}\text { Survival } \\
\text { interval }\end{array}$ & 1994-1995 & 1996-1997 & 1998-1999 & $2000-2001$ & $2002-2003$ & $p$ value \\
\hline \multirow[t]{12}{*}{ Metastatic } & \multirow[t]{4}{*}{ Overall } & $n$ & 364 & 345 & 409 & 514 & 564 & - \\
\hline & & 1-year & $37(32,42)$ & $34(29,39)$ & $41(36,46)$ & $46(42,51)$ & $52(48,57)$ & $<0.001$ \\
\hline & & 2-year & $16(12,19)$ & $14(10,17)$ & $19(15,23)$ & $22(18,26)$ & $28(24,4)$ & $<0.001$ \\
\hline & & 5 -year & $6(3,8)$ & $4(2,6)$ & $4(2,6)$ & $7(4,10)$ & $10(6,13)$ & 0.039 \\
\hline & \multirow[t]{4}{*}{ With chemotherapy } & $n$ & 94 & 110 & 159 & 227 & 289 & - \\
\hline & & 1-year & $63(53,73)$ & $52(42,61)$ & $57(50,65)$ & $70(63,76)$ & $75(70,80)$ & 0.016 \\
\hline & & 2-year & $29(19,38)$ & $24(16,32)$ & $27(20,34)$ & $34(27,41)$ & $41(35,47)$ & 0.015 \\
\hline & & 5 -year & $10(4,17)$ & $7(2,13)$ & $7(3,11)$ & $13(6,19)$ & $16(9,22)$ & 0.122 \\
\hline & \multirow[t]{4}{*}{ Without chemotherapy } & $n$ & 270 & 235 & 250 & 287 & 275 & - \\
\hline & & 1-year & $28(22,33)$ & $25(20,31)$ & $31(25,37)$ & $29(23,34)$ & $29(23,34)$ & 0.368 \\
\hline & & 2-year & $11(7,15)$ & $9(5,13)$ & $14(9,18)$ & $13(9,17)$ & $14(9,18)$ & 0.155 \\
\hline & & 5 -year & $4(2,7)$ & $3(<1,5)$ & $2(<1,5)$ & $3(1,6)$ & $4(1,7)$ & 0.428 \\
\hline \multirow[t]{12}{*}{ Non-metastatic } & \multirow[t]{4}{*}{ Overall } & $n$ & 1,567 & 1,670 & 1,814 & 2,005 & 2,075 & - \\
\hline & & 1-year & $91(89,92)$ & $90(88,92)$ & $90(88,91)$ & $90(89,92)$ & $93(91,94)$ & 0.040 \\
\hline & & 2-year & $82(80,85)$ & $83(8186)$ & $83(81,85)$ & $84(82,86)$ & $88(86,90)$ & $<0.001$ \\
\hline & & 5 -year & $71(68,74)$ & $71(68,74)$ & $70(67,73)$ & $71(68,73)$ & $77(74,79)$ & 0.002 \\
\hline & \multirow[t]{4}{*}{ With chemotherapy } & $n$ & 388 & 444 & 522 & 584 & 644 & - \\
\hline & & 1-year & $94(92,97)$ & $96(94,99)$ & $97(95,98)$ & $96(94,98)$ & $98(97,100)$ & 0.002 \\
\hline & & 2-year & $82(78,86)$ & $89(86,92)$ & $88(85,91)$ & $88(84,91)$ & $91(88,93)$ & $<0.001$ \\
\hline & & 5 -year & $63(58,69)$ & $71(66,76)$ & $71(67,76)$ & $69(64,73)$ & $76(72,80)$ & $<0.001$ \\
\hline & \multirow[t]{4}{*}{ Without chemotherapy } & $n$ & 1,179 & 1,226 & 1,292 & 1,421 & 1,431 & - \\
\hline & & 1-year & $89(87,91)$ & $88(86,90)$ & $87(85,89)$ & $88(86,90)$ & $90(88,92)$ & 0.373 \\
\hline & & 2-year & $82(80,85)$ & $81(79,84)$ & $81(78,84)$ & $82(80,85)$ & $87(84,89)$ & 0.011 \\
\hline & & 5 -year & $73(70,77)$ & $71(67,74)$ & $70(66,73)$ & $72(68,75)$ & $77(73,80)$ & 0.102 \\
\hline
\end{tabular}

(194 male and 134 female) of the 374 patients were included in this study according to the case selection criteria of this study. Furthermore, about half (46\%) of this study group were of age 70 years and older at the time of diagnosis. The major sites of the metastases were liver (239), lung (81), peritoneum (77), and non-regional lymph node (40). For the 328 patients in this review, the median length of time from date of diagnosis to the date of death or the last follow-up was 11.9 months, ranging from less than a month to 33.8 months. At the end of follow-up, 253 (77\%) patients had died with 229 (91\%) of them dying from colorectal cancer. The causes of death for the remaining 24 patients were due to another cancer or anything not related to cancer; these were censored in the cause-specific survival analysis.

In total, 255 of the 328 patients had their first appointment for any treatment in a cancer treatment center in Alberta. Among 73 patients who did not have treatment appointments in the cancer treatment centers, 69 had died within 2 months. The remaining four patients died from other or unknown causes. When the total 255 patients were followed from the date of the first appointment of any treatment to the date of death/last follow-up, the median OS was 18.2 months for patients aged under 70 compared to 10.2 months for patients over 70 year of age $(p<0.0036)$. Patients aged under 70 were more likely to receive chemotherapy than patients aged over 70 (Table 2).

A total of 168 of the 255 patients received chemotherapy. Results from the multivariate analysis using Cox models have shown that chemotherapy significantly improved the chance of survival $(\mathrm{HR}=0.31,95 \% \mathrm{CI}=0.21-0.44)$. The hazard ratio (HR) of 0.31 represents a $69 \%$ reduction in the risk of mortality. In contrast, being over the age of $70(\mathrm{HR}=1.44$, $95 \% \mathrm{CI}=1.00-2.07)$ or having more than one distant metastatic sites significantly reduced the chances of survival ( $\mathrm{HR}=$ 2.27, 95\% CI $=1.62-3.19$ ) (Table 3). When the 168 patients treated with chemotherapy were followed from the date of their first chemotherapy appointment to the date of death/last follow-up, the median OS was 17.5 months $(95 \% \mathrm{CI}=14.3$ 21.3) (Fig. 1). Among this group, patients aged over 70 had a significantly lower chance of survival than patients aged under 70 years of age $(p=0.0031)$. 
Table 2 Median overall survival (OS) for metastatic colorectal cancer cases diagnosed in Alberta, 2004

\begin{tabular}{lccc}
\hline Factors & From first treatment appointment to the last follow-up & \\
\cline { 2 - 4 } & Chemo/total (\%) 168/255 (66\%) & Median OS (in months) $(95 \%$ CI) & $p$ value \\
\hline Gender & & & \\
Male & $93 / 149(62 \%)$ & $13.5(11.2-17.1)$ & 0.398 \\
Female & $75 / 106(71 \%)$ & $16.1(10.5-20.8)$ & \\
Age & $42 / 91(46 \%)$ & & 0.004 \\
70 years and older & $126 / 164(77 \%)$ & $10.2(6.7-12.9)$ & \\
Less than 70 years & $37 / 60(62 \%)$ & $18.2(15.0-22.1)$ & \\
Number of malignancies & $131 / 195(67 \%)$ & $12.2(6.7-19.0)$ & 0.140 \\
2 or more & & $15.0(12.8-19.1)$ & \\
1 & $56 / 89(63 \%)$ & $9.2(5.4-12.0)$ & \\
Number of metastases & $112 / 166(67 \%)$ & $19.8(15.0-26.6)$ & \\
2 or more & & $14.3(10.9-17.7)$ & 0.001 \\
1 & $120 / 181(66 \%)$ & $15.6(11.5-32.5)$ & \\
Metastatic sites & $48 / 74(65 \%)$ & & \\
Hepatic & &
\end{tabular}

\section{Discussion}

The proportion of patients with metastatic colorectal cancer treated with chemotherapy has doubled in the past 10 years. Throughout the same period, we have also seen a significant increase in survival rates for patients who received chemotherapy, more for 1-year and 2-year survival than for 5-year survival. In addition to the evaluation of trends in relative survival over a 10 -year period, patients with metastatic colorectal cancer diagnosed in 2004 were also reviewed. We saw a $69 \%$ reduction in the risk of mortality in patients who received chemotherapy compared to those who did not receive chemotherapy. The median OS of patients who received chemotherapy was 17.5 months.
This was similar to the median OS of 18-20 months, based on a review of recently published clinical trial studies. Further investigation is required in order to determine whether such improvement was due to the increased use of chemotherapy or advances made in the efficacy of chemotherapy drugs.

There are several strengths of the present study. Patients included in this study were identified from a populationbased cancer registry and included almost all patients diagnosed in Alberta in the past 11 years. Results from this study should represent the cancer patient population in Alberta as a whole. Improvements in survival rates over time have often been used as an indicator for advances made in cancer treatment. However, survival rates may also
Table 3 Hazard ratios (HR) of mortality for patients with metastatic colorectal cancer in Alberta, 2004

\begin{tabular}{llll}
\hline \multirow{2}{*}{ Factors } & \multicolumn{2}{l}{ From first treatment appointment to the last follow-up } \\
\cline { 2 - 4 } & $\begin{array}{l}\text { Case } \\
N=255\end{array}$ & $\begin{array}{l}\text { Unadjusted HR } \\
(95 \% \mathrm{CI})\end{array}$ & $\begin{array}{l}\text { Adjusted HR } \\
(95 \% \mathrm{CI})\end{array}$ \\
\hline Chemo & $168(66 \%)$ & $0.31(0.22-0.44)$ & $0.31(0.21-0.44)$ \\
Yes & $87(34 \%)$ & 1.00 & 1.00 \\
No & $91(36 \%)$ & $1.67(1.20-2.31)$ & $1.44(1.00-2.07)$ \\
Age & $164(64 \%)$ & 1.00 & 1.00 \\
70 years and older & & & \\
Less than 70 years & $89(35 \%)$ & $2.03(1.47-2.81)$ & $2.27(1.62-3.19)$ \\
Metastases & $166(65 \%)$ & 1.00 & 1.00 \\
\# Sites & & & $1.33(0.91-1.94)$ \\
2 or more & $181(71 \%)$ & $1.37(0.96-1.97)$ & 1.00 \\
1 & $74(29 \%)$ & 1.00 & \\
Site & & & \\
Hepatic & &
\end{tabular}


Fig. 1 Median overall survival (OS) from the first chemotheraphy

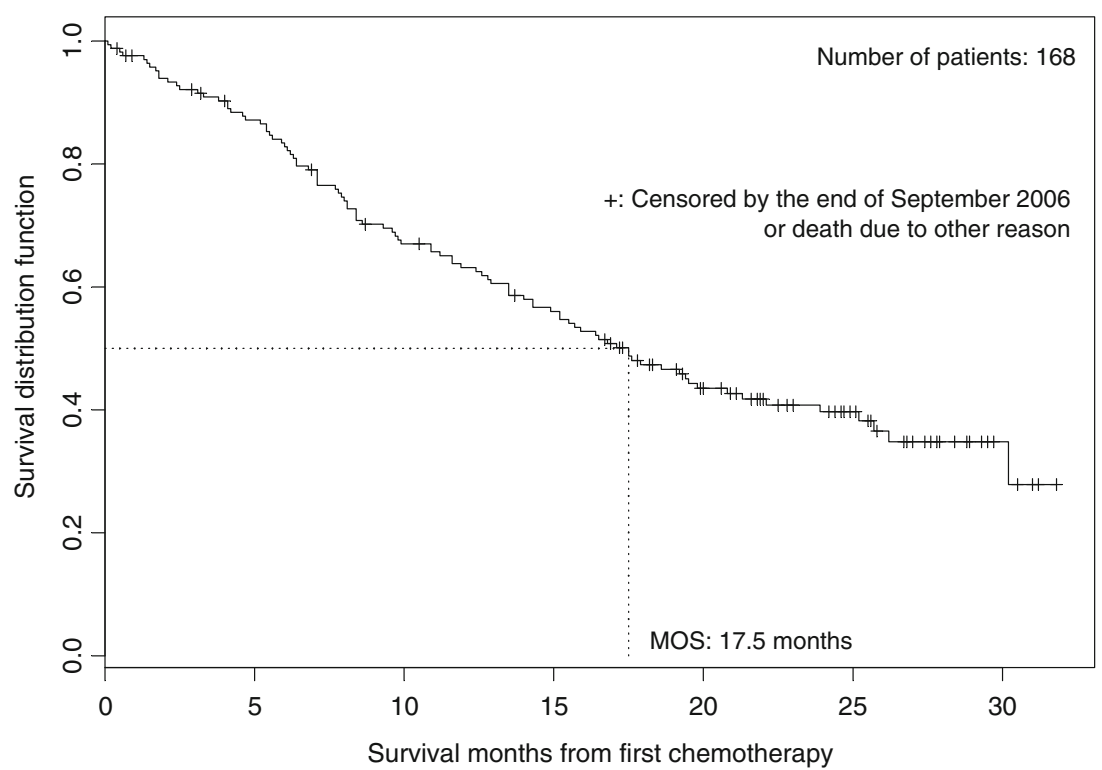

improve if cancers are detected at an earlier stage, even if no progress has been made in cancer treatments. Without stage-specific survival information, interpretation of the improved survival observed is limited [8]. The current study was able to focus on the survival of patients with latestage colorectal cancer for which chemotherapy is the main treatment modality. Observed improvements in the survival of patients with metastatic colorectal cancer, therefore, were probably due to chemotherapy treatment. Previous studies based on large population-based cancer registry data in Europe and in the USA have shown that the survival of colorectal cancer varies with morphology type [19]. For example, Carcinoid had a 5-year relative survival rate of $83 \%$ compared to $62 \%$ for overall colorectal cancer. In addition, cases with a microscopically confirmed diagnosis generally displayed better survival than those which did not. The 5 -year survival rate was only $9 \%$ for cases with no microscopically confirmed diagnoses. In order to monitor the survival trends accurately, this study has only included microscopically confirmed adenocarcinoma cases which comprised $90 \%$ of the colorectal cancer cases diagnosed in the study period.

Although there are strengths of the present study, there are also limitations. Greater survival rates observed in patients who received chemotherapy, in part, can be attributed to the selection of healthier patients into the treatment group. Although we assessed the association between chemotherapy and survival using multivariate analysis to account for the differences in age, gender, and characteristics of the metastasis between groups of patients who received and who did not receive chemotherapy, many factors influencing the chemotherapy decisions and survival outcomes are difficult to measure and control completely. These confounding factors may include socio-economic factors and patients' health status. Patient preferences may also have impacted the chemotherapy decision, but we were not able to capture this information from the existing EMR cancer clinical database. Additional data linkage with the provincial health administrative database that covers a wide range of health services activities provided to Albertans may offer opportunities to collect further information on comorbidities of the cancer patients. Due to this limitation, the study was not able to establish a direct causal relationship but to suggest an association between the observed survival improvement of the metastatic colorectal cancer patients and the chemotherapy treatment that they received.

Only median OS was measured using the existing clinical database in this study. Other indicators, such as time-toprogression and progression-free survival, can provide better outcome information for a specific treatment but they cannot be measured directly in the present EMR system. This is because the information on progression/recurrence is only routinely available from physicians' dictated notes and not in a format that can be extracted electronically. A manual review would be required to obtain this information. Another way to obtain progression information is based on changes in chemotherapy drugs in a patient, but this method can only give imprecise assumptions on progression. Many patients with earlier stage colorectal cancer will develop metastatic disease due to progression after their initial treatments. Because of not being able to capture cancer progression data electronically, we presently cannot identify these patients or include them in the outcome evaluation. In addition, data from the EMR is patient specific but not cancer specific; therefore, the linkage between the Cancer Registry and the EMR is not a straightforward process for patients who had more than one primary cancer. Improvements in linkage techniques or 
in the linkage of treatment information to specific cancers could improve the quality of the linked data.

In the current study, using data from the EMR, the median OS was 17.5 months for a cohort of patients who were diagnosed in 2004 and received chemotherapy in Alberta. This was quite close to the 18 months achieved in clinical trials. However, because of selection bias, survival results from our observational cohort cannot be compared directly with that from the randomized clinical trials. Most of the published evidence on the efficacy of a treatment is based on well-controlled randomized clinical trials that are usually conducted among selected patients. Specific selection criteria are used to ensure that treatment and control groups are similar in relation to the distribution of factors influencing the treatment outcomes [20]. Patients over 70 years of age are often under-represented in these treatment trials [21]. In contrast, our study included patients in real clinical care environments, and nearly half of them were older than 70; many of them might have important comorbidities. Median OS of these patients seen in clinical practice may have been worse than that for the patients selected for clinical trials. Nevertheless, this study has demonstrated the applicability of randomized clinical trial data in the evaluation of treatment outcomes in clinical practice.

The focus of this population-based study was to investigate the survival for patients who were given chemotherapy in a representative patient population in Alberta. This study has demonstrated an opportunity to conduct ongoing evaluation and monitoring of outcomes using a population-based data set established by linking the Cancer Registry data with an EMR clinical database. The results from these ongoing evaluations can contribute to clinical decision making and to policy development [22]. The priorities in developing this ongoing system include accurately linking Cancer Registry data and the data from the EMR by patient and diagnosis, capturing cancer recurrence and cancer progression information, identifying key outcome indicators and associated data elements that are routinely collected in the EMR, and improving the quality of the key data elements used in the treatment outcome evaluation.

Chemotherapy given to patients who had diagnosed with metastatic cancer or had recurrent cancer is palliative chemotherapy. When considering the treatment and care for patients whose cancers have developed to an advanced stage, the type of supportive care and the quality of life of the patients are as important as survival improvement.

\section{Conclusions}

There was an improvement in the survival of metastatic colorectal cancer patients in recent years in Alberta and this improvement is most likely attributable in large part to the chemotherapy treatment that they received.

Open Access This article is distributed under the terms of the Creative Commons Attribution Noncommercial License which permits any noncommercial use, distribution, and reproduction in any medium, provided the original author(s) and source are credited.

\section{References}

1. Alberta Cancer Board Cancer in Alberta (2007) A Regional Picture 2007. Alberta Cancer Board, Alberta

2. Ellison LF, Gibbons L (2006) Survival from cancer-up-to-date predictions using period analysis. Health Rep 17(2):19-30

3. Canadian Cancer Society/National Cancer Institute of Canada (2007 Jan 4) Canadian Cancer Statistics 2007. Canadian Cancer Society, Toronto

4. Canadian Task Force on Preventive Health Care (2001) Colorectal cancer screening; Recommendation statement from the Canadian Task Force on Preventive Health Care. CMAJ 165(2):206-208

5. McGregor SE, Hilsden RJ, Li FX, Bryant HE, Murray A (2007) Low uptake of colorectal cancer screening $3 \mathrm{yr}$ after release of national recommendations for screening. Am J Gastroenterol 102 (8):1727-1735. doi:10.1111/j.1572-0241.2007.01217.x

6. American Joint Committee on Cancer (2002) AJCC Cancer Staging Manual, 6th edn. Springer, New York

7. Ries LA, Wingo PA, Miller DS et al (2000) The annual report to the nation on the status of cancer, 1973-1997, with a special section on colorectal cancer. Cancer 88(10):2398-2424. doi:10.1002/(SICI)1097-0142(20000515)88:10<2398::AIDCNCR26>3.0.CO;2-I

8. O'Connell JB, Maggard MA, Ko CY (2004) Colon cancer survival rates with the new American Joint Committee on Cancer sixth edition staging. J Natl Cancer Inst 96(19):1420 1425

9. Pasetto LM, Jirillo A, Iadicicco G, Rossi E, Paris MK, Monfardini S (2005) FOLFOX versus FOLFIRI: a comparison of regimens in the treatment of colorectal cancer metastases. Anticancer Res 25 (1B):563-576

10. Saletti P, Cavalli F (2006) Metastatic colorectal cancer. Cancer Treat Rev 32(7):557-571. doi:10.1016/j.ctrv.2006.07.005

11. Goyle S, Maraveyas A (2005) Chemotherapy for colorectal cancer. Dig Surg 22(6):401-414. doi:10.1159/000091441

12. Potosky AL, Riley GF, Lubitz JD, Mentnech RM, Kessler LG (1993) Potential for cancer related health services research using a linked Medicare-tumor registry database. Med Care 31(8):732748. doi:10.1097/00005650-199308000-00006

13. Edwards BK, Brown ML, Wingo PA et al (2005) Annual report to the nation on the status of cancer, 1975-2002, featuring population-based trends in cancer treatment. J Natl Cancer Inst 97(19): 1407-1427

14. Fritz A, Percy C, Jack A, Shanmugaratnam K, Sobin LH, Parkin MD (2000) International classification of diseases for oncology (ICD-O), 3rd edn. World Health Organization, Geneva

15. Havener L (ed) (2004) Standards for Cancer Registries; Vol III; Standards for completeness, quality, analysis, and management of data. Springfield, IL, North American Association of Central Cancer Registries, Inc.

16. Parkin DM, Hakulinen T (1991) Chapter 12. Analysis of survival. In: Jensen OM, Parkin DM, MacLennan R, Muir CS, Skeet RG (eds) Cancer registry: principles and methods. International Agency for Research on Cancer, Lyon, pp 159-176 
17. Brenner H, Gefeller O (1996) An alternative approach to monitoring cancer patient survival. Cancer 78(9):2004-2010. doi:10.1002/(SICI) 1097-0142(19961101)78:9<2004::AID-CNCR23>3.0.CO;2-\#

18. Ederer F, Axtell LM, Cutler SJ (1961) The relative survival rate: a statistical methodology. Natl Cancer Inst Monogr 6:101-121

19. Gatta G, Ciccolallo L, Capocaccia R et al (2003) Differences in colorectal cancer survival between European and US populations: the importance of sub-site and morphology. Eur J Cancer 39 (15):2214-2222. doi:10.1016/S0959-8049(03)00549-5
20. Miller KD, Rahman ZU, Sledge GW Jr (2001) Selection bias in clinical trials. Breast Dis 14:31-40

21. Talarico L, Chen G, Pazdur R (2004) Enrollment of elderly patients in clinical trials for cancer drug registration: a 7-year experience by the US Food and Drug Administration. J Clin Oncol 22(22):4626-4631. doi:10.1200/JCO.2004.02.175

22. Sharpe N (2002) Clinical trials and the real world: selection bias and generalisability of trial results. Cardiovasc Drugs Ther 16 (1):75-77. doi:10.1023/A:1015327801114 